\title{
SISTEMA AUTOMÁTICO DE MEDIÇÃO DE RUÍDO MAGNÉTICO BARKHAUSEN*
}

\section{Resumo}

Roger Ferreira da Silva ${ }^{1}$ Silvério Ferreira da Silva Júnior ${ }^{2}$ Renato Cardoso Mesquita ${ }^{3}$ Marina Campos Oliveira Silva

O método de ensaio não destrutivo baseado na análise do ruído magnético Barkhausen (RMB) possibilita a obtenção de informações a respeito da microestrutura do material examinado e do estado de tensões presente no mesmo. Devido às particularidades do método, as medições devem ser realizadas mantendo-se constante a geometria entre a sonda e o objeto examinado, de forma a assegurar a confiabilidade das mesmas. Quando realizado manualmente, 0 processo de medição é demorado e sujeito a erros de posicionamento da sonda. Em trabalhos industriais ou em laboratório, esses erros podem resultar em interpretações incorretas, devendo ser reduzidos ao mínimo possível. Visando à redução do tempo de execução das medições e de análise e a eliminação de possíveis erros de posicionamento da sonda de inspeção, este trabalho propõe a automação do processo de medição de RMB em corpos de prova do tipo chapa. Compreende a construção de um dispositivo de movimentação $\mathrm{XYZ}$ acionado por motores de passo e do software "Plate Barkhausen", com uma interface amigável, para controle da movimentação e da medição, além da análise dos dados.

Palavras-chave: Ensaio não destrutivo; Ensaio magnético; Barkhausen; Automação

\begin{abstract}
AUTOMATIC SYSTEM FOR MEASUREMENT OF BARKHAUSEN NOISE Abstract

The test method based on the magnetic Barkhausen noise analysis allows us to get information about the microstructure of the examined material and its stress state. Due to the method features, the measurements must be realized keeping the geometry between the probe and the examined object constant, to ensure the reliability of them. When realized manually, the measuring process spends much time and is subjected to probe positioning errors. In industrial works or in laboratory, those errors can result in incorrect interpretation and should be kept to a minimum. Aiming to the reduction of the time of measurements execution and of analysis and the elimination of possible probe positioning errors, this work proposes the automation of the measuring process of magnetic Barkhausen noise in specimens of plate type. It consists in a XYZ moving dispositive and the "Plate Barkhausen" software for control of the moving and measuring, as well the data analysis.
\end{abstract}

Keywords: Nondestructive testing; Magnetic testing; Barkhausen; Automation

1 Engenheiro de Controle e Automação, Mestrando em Ciência e Tecnologia das Radiações e Minerais e Materiais, Técnico, Serviço de Integridade Estrutural, Centro de Desenvolvimento da Tecnologia Nuclear, Belo Horizonte, MG.

2 Engenheiro Mecânico, Doutor em Tecnologia Nuclear, Tecnologista Senior, Serviço de Integridade Estrutural, Centro de Desenvolvimento da Tecnologia Nuclear, Belo Horizonte, MG.

3 Engenheiro Eletricista, Doutor em Engenharia Elétrica, Professor Titular, Departamento de Engenharia Elétrica, Universidade Federal de Minas Gerais, Belo Horizonte, MG.

4 Estudante Engenharia Mecânica, CEFET-MG, Belo Horizonte, MG.

* Contribuição técnica ao $18^{\circ}$ Seminário de Automação e TI Industrial, 23 a 26 de setembro de 2014, São Paulo, SP, Brasil. 


\section{INTRODUÇÃO}

Os Ensaios Não Destrutivos são técnicas utilizadas na inspeção de materiais e equipamentos sem danificá-los, sendo executados nas etapas de fabricação, construção, montagem e manutenção. Constituem uma das principais ferramentas do controle da qualidade de materiais e produtos, sendo largamente utilizados nos setores nuclear, petróleo/petroquímico, químico, aeronáutico, aeroespacial, siderúrgico, naval, entre outros.

No Laboratório de Ensaios Não Destrutivos (LABEND) do Centro de Desenvolvimento da Tecnologia Nuclear (CDTN), são desenvolvidas pesquisas a respeito do uso do método de ensaio de materiais baseado na análise do Ruído Magnético Barkhausen (RMB). Esse fenômeno consiste em mudanças abruptas da magnetização do material quando submetido a um campo magnético variável, devidas à resistência ao movimento das paredes de domínio. Ensaios Não Destrutivos baseados na análise do RMB podem ser usados para a avaliação de materiais ferromagnéticos quanto à sua microestrutura e estado de tensões [1] e são muito adotados em indústrias, como a automotiva. Características como tamanho de grão, dureza, quantidade de precipitados, danos induzidos por fadiga e a presença de tensões residuais podem ser avaliadas. Na indústria nuclear, este método de ensaio tem sido utilizado em trabalhos de pesquisa para a avaliação de danos produzidos em materiais estruturais expostos a feixes de nêutrons.

Para a realização das medições de RMB, o LABEND dispõe do medidor digital de RMB modelo Rollscan 300 da fabricante Stresstech, suas sondas de exame e o software "MicroScan 600" para aquisição de dados instalado em um computador. Para as medições, o operador deve posicionar a sonda sobre o objeto a ser examinado e, então, acionar o comando para a medição e aquisição dos dados pelo software "MicroScan 600". A sonda aplica ao material um campo magnético (excitação) e, ao mesmo tempo, mede os sinais referentes ao RMB produzido no material. Após a aquisição, esses dados brutos são analisados pelo mesmo programa - novamente a pedido do operador - e os resultados da análise são usados pelo operador para o diagnóstico a respeito do material. Esse processo pode demorar e, normalmente, é repetido para vários pontos da superfície, o que o torna cansativo e repetitivo. Além disso, o posicionamento da sonda e a força com que é pressionada contra o objeto influenciam na medida, logo, o operador deve estar atento a eles.

A automação de processos, sejam industriais ou laboratoriais, pode ser utilizada para agilizar a execução de atividades como a descrita anteriormente, facilitar o controle e minimizar a variabilidade e os desvios dos processos [2], além de diminuir o esforço humano. O sucesso do uso de computadores para controlar processos complexos foi alcançado primeiramente no ambiente industrial, e, com o tempo, o desenvolvimento tecnológico e a automação chegaram aos laboratórios de pesquisa. Há algumas diferenças entre o controle de processos industriais e laboratoriais: primeiramente, nos laboratórios, há muito menos ênfase em confiabilidade e redundância dos componentes, uma vez que a indisponibilidade de um equipamento é muito menos custosa em um laboratório do que em uma indústria de grande produção; além disso, automação laboratorial demanda maior flexibilidade, uma vez que, em pesquisa científica, procedimentos podem mudar diariamente ou semanalmente, o que não é comum acontecer em indústrias [3].

No âmbito da automação, motores de passo são uma boa opção para posicionamento. Fontana [4] e Sato [5] usam motores de passo em seus trabalhos.

* Contribuição técnica ao $18^{\circ}$ Seminário de Automação e TI Industrial, 23 a 26 de setembro de 2014, São Paulo, SP, Brasil. 
Fontana considera esse motor a melhor opção para o controle automático de posição para telescópios, já que o posicionamento do motor de passo não sofre tanto com interferências externas, podendo ser usado em malha aberta, sem qualquer realimentação de controle. Já Sato utiliza motores de passo para o controle de abertura e fechamento de válvulas em um sistema automático de carregamento em ensaios estruturais. Segundo ele, os motores de passo possuem total adaptação por controles digitais, além de serem muito precisos. Contudo, possuem má relação potência-volume.

Devido às particularidades do método de RMB, as medições devem ser realizadas mantendo-se constante a geometria entre a sonda e o objeto examinado, de forma a assegurar a confiabilidade das mesmas. Quando realizado manualmente, 0 processo de medição é demorado e sujeito a erros de posicionamento da sonda. Em trabalhos industriais ou em laboratório, esses erros podem resultar em interpretações incorretas, devendo ser reduzidos ao mínimo possível. A automação das medições de RMB é necessária para garantir a precisão do deslocamento da sonda e do posicionamento da mesma em medições que devem ser repetidas periodicamente [6].

É proposta, então, a implementação do Sistema Automático de Medição de Ruído Magnético Barkhausen (SAMRMB) em corpos de prova do tipo chapa, visando à redução do tempo de execução das medições e de análise e à padronização das operações de medição, eliminando possíveis erros de posicionamento da sonda e variações na força com que a sonda é mantida na superfície em exame e, consequentemente, melhorando a repetibilidade de resultados. O SAMRMB é constituído por software e hardware.

\section{MATERIAIS E MÉTODOS}

O Sistema Automático de Medição de Ruído Magnético Barkhausen (SAMRMB) realiza medições de RMB em chapas utilizando o medidor digital de RMB Rollscan 300. Neste trabalho, são desenvolvidos dois módulos do sistema:

- O dispositivo de movimentação XYZ responsável pelo posicionamento da sonda do medidor sobre a superfície da chapa em exame.

- O software "Plate Barkhausen" para controle do posicionamento da sonda e controle da medição de RMB, além da aquisição e análise de dados.

O esquema funcional do SAMRMB está apresentado na Figura 1.

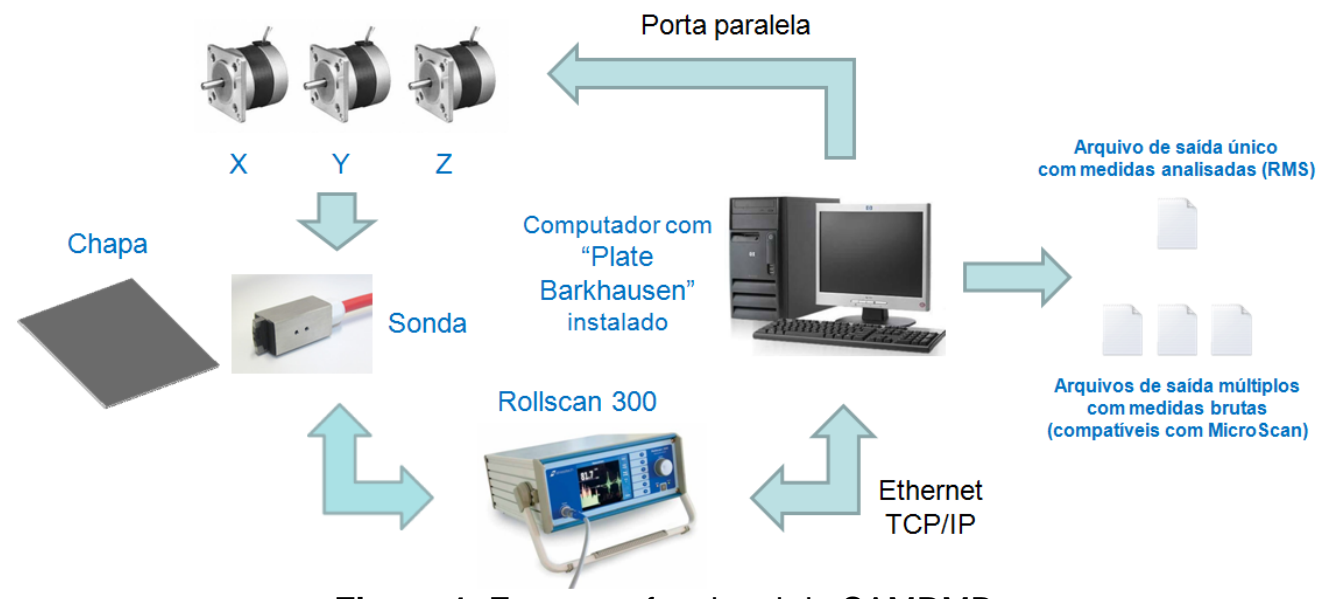

Figura 1. Esquema funcional do SAMRMB.

* Contribuição técnica ao $18^{\circ}$ Seminário de Automação e TI Industrial, 23 a 26 de setembro de 2014 , São Paulo, SP, Brasil. 
O dispositivo de movimentação $X Y Z$ responsável pelo posicionamento da sonda do Rollscan 300 sobre a superfície da chapa possui três motores de passo, um para cada eixo de movimento. O medidor é controlado pelo software "Plate Barkhausen" (instalado no computador), que, através de comunicação TCP/IP (rede Ethernet), envia comandos de medição e configuração ao equipamento e recebe os valores das medidas. O controle da movimentação dos motores é feito pelo "Plate Barkhausen" através da porta paralela do computador. O software analisa os dados lidos do Rollscan 300 e exporta os dados brutos e o resultado da análise.

\subsection{0 software "Plate Barkhausen"}

O "Plate Barkhausen" é a interface entre o usuário e o SAMRMB e centraliza todo o controle do sistema (controle do medidor Rollscan 300, controle de posicionamento e análise de dados). Desenvolvido no ambiente Microsoft Visual $C_{++} 2010$, o software possui 16 janelas e duas bibliotecas. A biblioteca "Rollscan" encapsula o código referente à comunicação entre o computador e o medidor via socket TCP/IP. Para a obtenção do protocolo de comunicação do Rollscan 300 , foi feito contato com a fabricante Stresstech. Como a Stresstech possui outros modelos de medidores digitais de RMB com protocolos de comunicação similares (exceto pela presença de novos comandos ou ausência de outros), essa biblioteca pode ser usada para a operação de outros modelos além do Rollscan 300, bastando, se necessário, efetuar pequenas modificações em seu código.

A biblioteca "PortaParalela" permite o acesso à porta paralela do computador de forma facilitada. A porta paralela é usada para o controle dos motores de passo do dispositivo XYZ.

Na Figura 2, é apresentada a janela principal do software. Nela, pode ser visto o menu "Controle" aberto e o gráfico de uma aquisição realizada, com o sinal de excitação senoidal e o RMB.

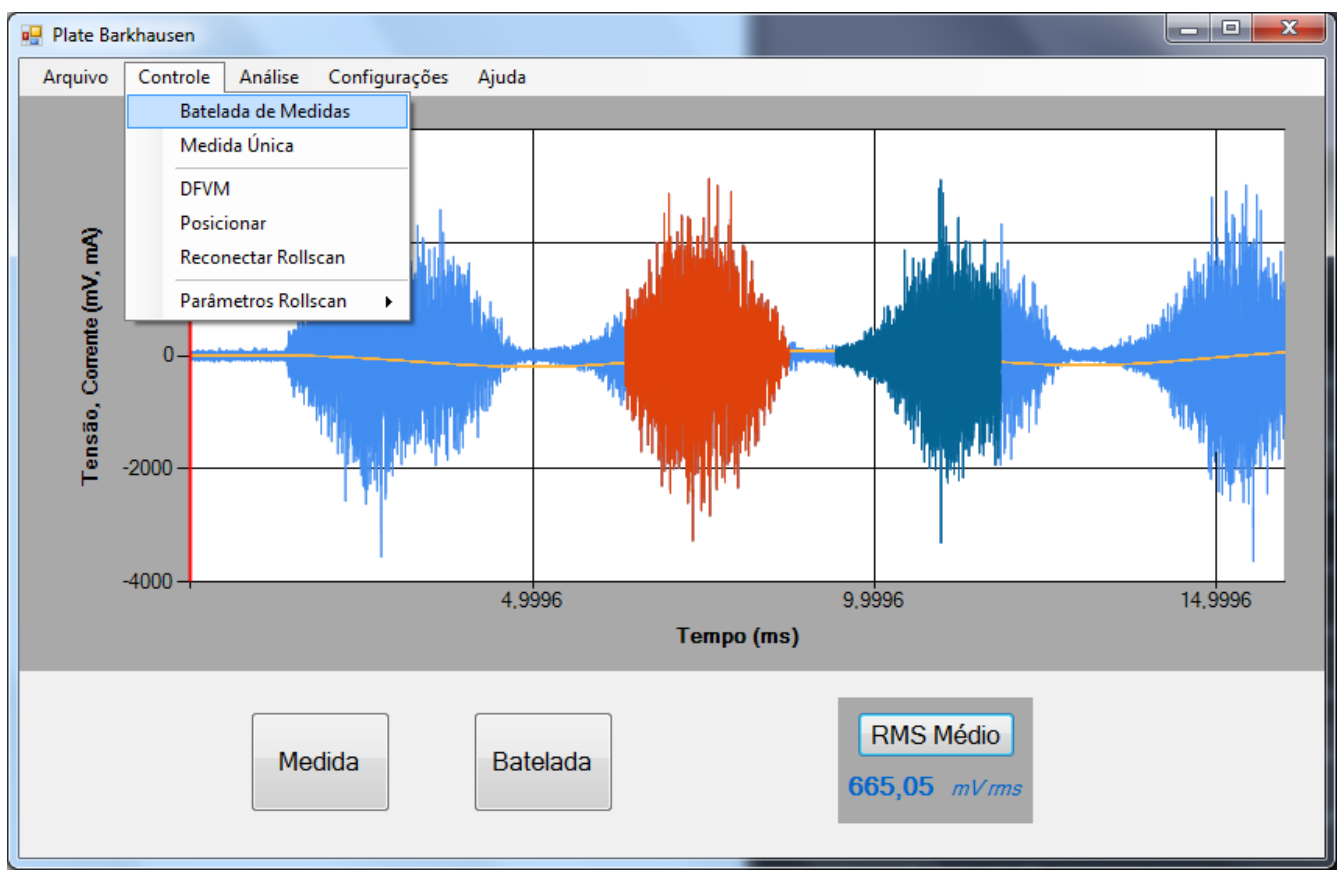

Figura 2. Janela principal do "Plate Barkhausen". Menu "Controle" aberto e gráfico de uma aquisição realizada com destaque para os bursts centrais, que são usados para o cálculo do valor RMS.

Por meio dos botões e menus da tela principal, é possível:

* Contribuição técnica ao $18^{\circ}$ Seminário de Automação e TI Industrial, 23 a 26 de setembro de 2014, São Paulo, SP, Brasil. 
- Alterar os parâmetros do Rollscan 300, como a forma de onda de magnetização (Figura 3);

- Posicionar a sonda do medidor;

- Executar aquisição única de RMB;

- Executar batelada automática de aquisições de RMB (variando os parâmetros ou a posição do sensor entre cada medida);

- Obter o valor RMS (Root Mean Square) do sinal adquirido;

- Guardar os dados em arquivo para posterior visualização.

Figura 3. Janela "Forma de Onda". Permite selecionar a forma de onda de magnetização do medidor.

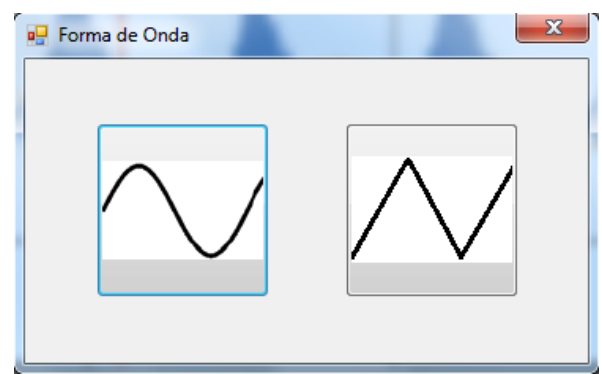

Para efetuar uma aquisição única ou batelada de aquisições de RMB, é necessário:

- Configurar os parâmetros da medição (frequência e voltagem de magnetização, trajetória da batelada, arquivo de exportação de dados, entre outros) por meio da janela "Configuração de Medição" (Figuras 4 e 5). A aba "Batelada" (Figura 5) não se aplica à aquisição única ou DFVM;

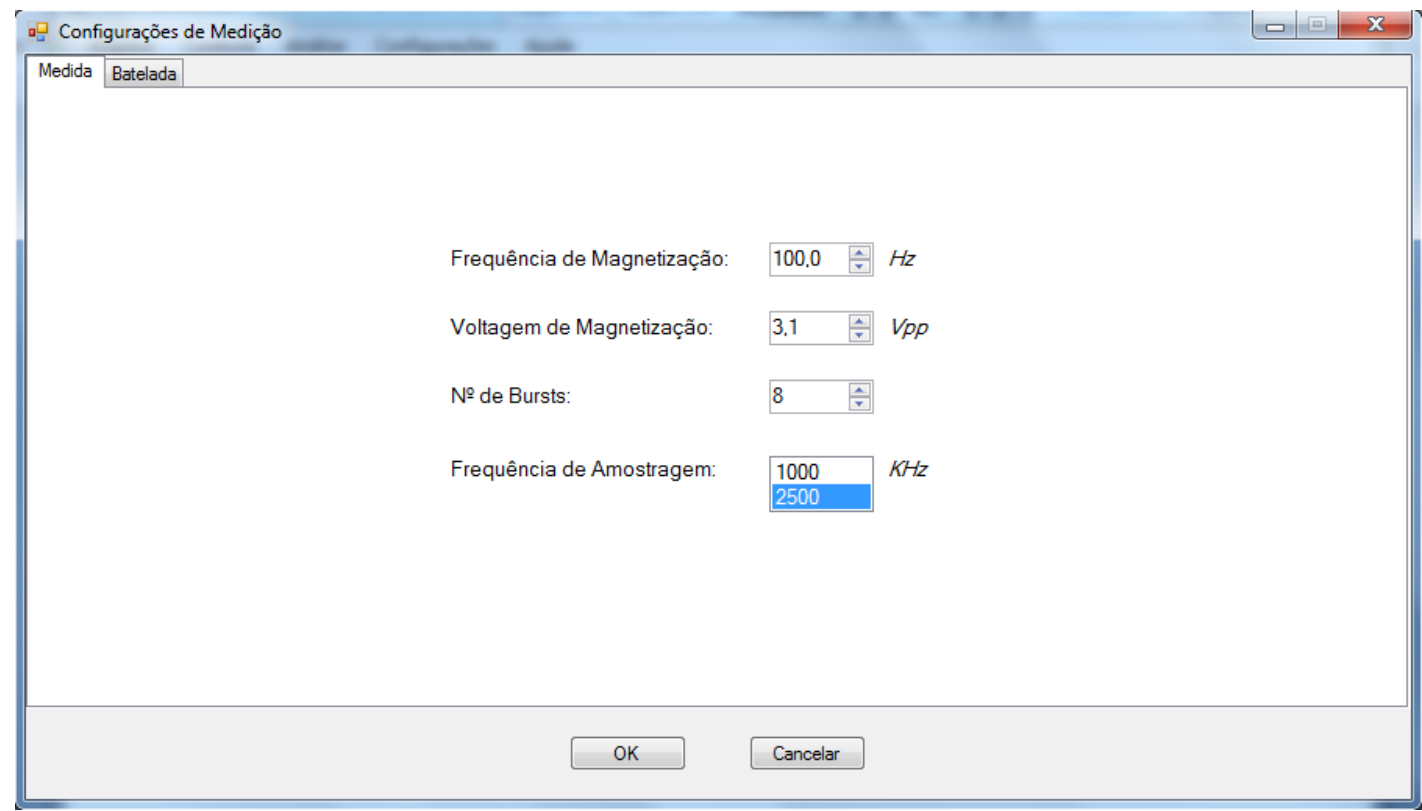

Figura 4. Aba "Medidas" da janela "Configurações de Medição". Permite a configuração dos parâmetros da medida.

* Contribuição técnica ao $18^{\circ}$ Seminário de Automação e TI Industrial, 23 a 26 de setembro de 2014, São Paulo, SP, Brasil. 


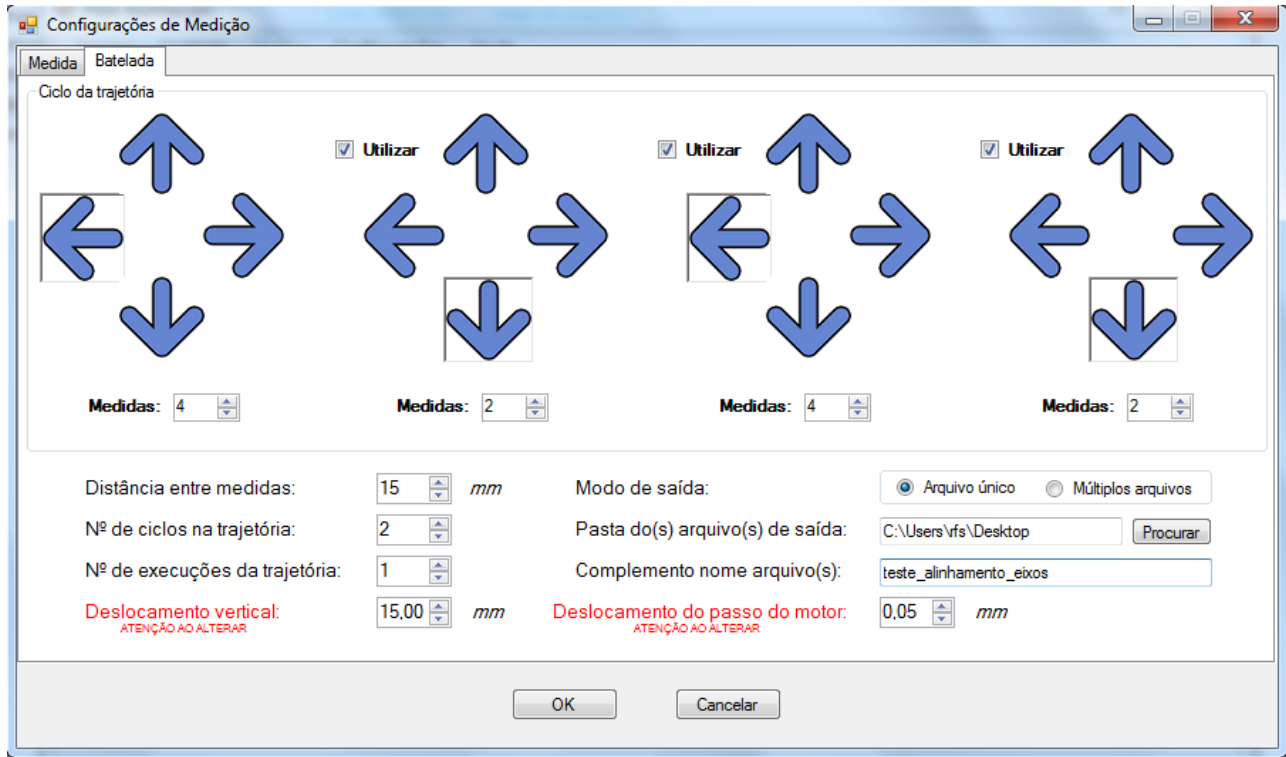

Figura 5. Aba "Batelada" da janela "Configurações de Medição". Permite a configuração dos parâmetros da batelada de aquisições.

- Posicionar a sonda no ponto desejado da peça para a aquisição única, para DFVM ou início da trajetória da batelada, por meio do Modo de Posicionamento (Figura 6);

- Executar, então, a medição por meio dos botões da janela principal.

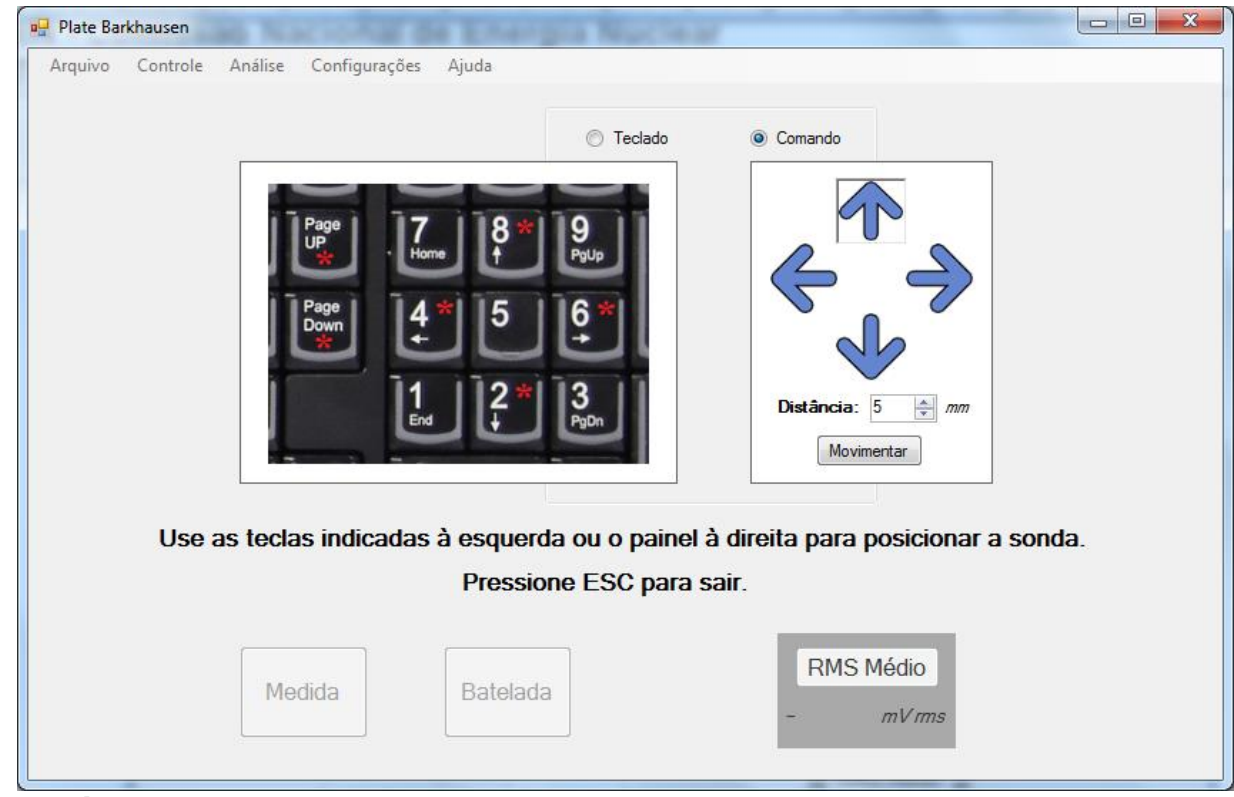

Figura 6. Modo de Posicionamento. Permite o posicionamento da sonda.

Na batelada com movimentação, o sensor é movimentado de acordo com a trajetória configurada, sendo aproximado e afastado da chapa em exame a cada aquisição realizada. Durante o processo de batelada, ainda, é criado um arquivo de saída com o valor RMS (média dos valores RMS dos bursts centrais) do sinal adquirido em cada ponto da trajetória e arquivos múltiplos com as medidas brutas em cada ponto. $\mathrm{Na}$ batelada sem movimentação do sensor, frequência e voltagem de magnetização são variadas entre cada medida dentro de intervalos configurados no modo DFVM (Determinação da frequência e voltagem de magnetização) (Figura 7), e são gerados também os arquivos de saídas citados anteriormente.

* Contribuição técnica ao $18^{\circ}$ Seminário de Automação e TI Industrial, 23 a 26 de setembro de 2014, São Paulo, SP, Brasil. 

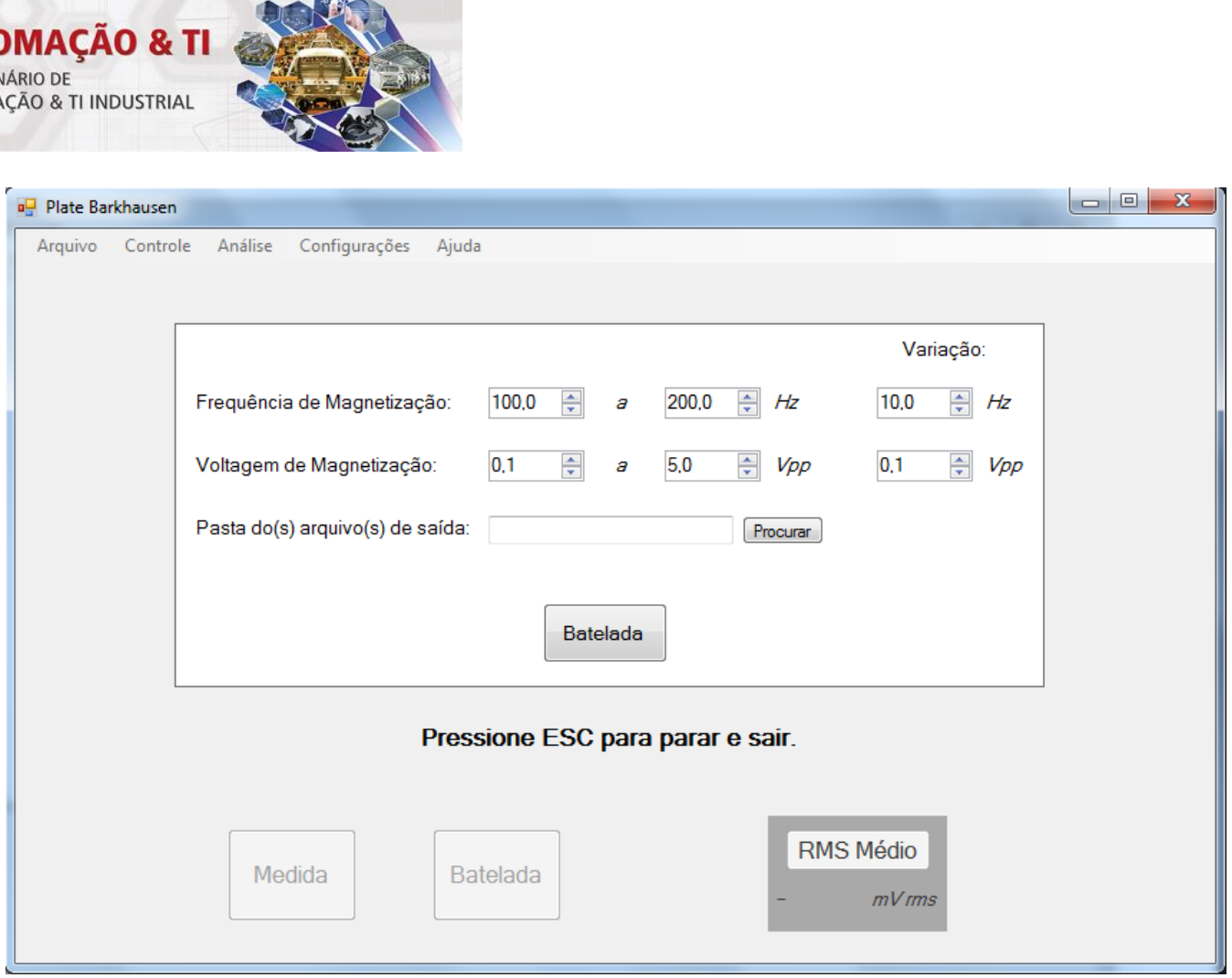

Figura 7. Modo DFVM. Permite executar batelada sem movimentação do sensor, mas variando frequência e voltagem de magnetização.

Após uma aquisição única, o botão "RMS Médio" da tela principal permite calcular o valor RMS do sinal coletado (média dos valores RMS bursts centrais).

\subsection{O Dispositivo XYZ}

Antes do projeto do dispositivo $X Y Z$, foi feita uma pesquisa das práticas usuais na construção de sistemas de movimentação com motor de passo. Além disso, foram verificados os componentes existentes no mercado para a construção do dispositivo. O desenho simplificado do dispositivo pode ser visto na Figura $8 \mathrm{a}$, com destaque para a mesa onde é colocada a placa a ser inspecionada, os eixos e o sensor. $O$ dispositivo já construído é apresentado na Figura 8b.

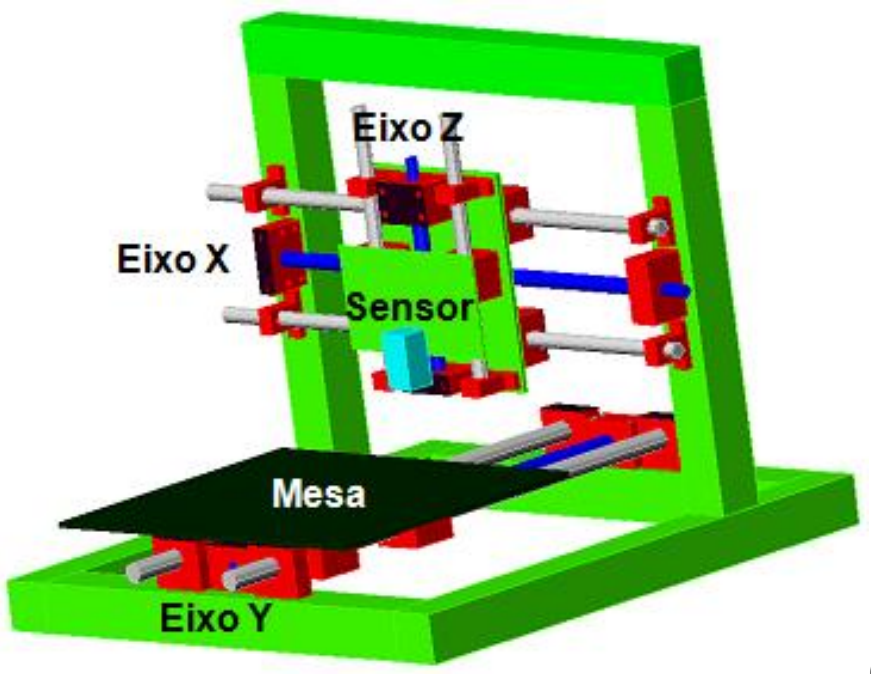

(a)

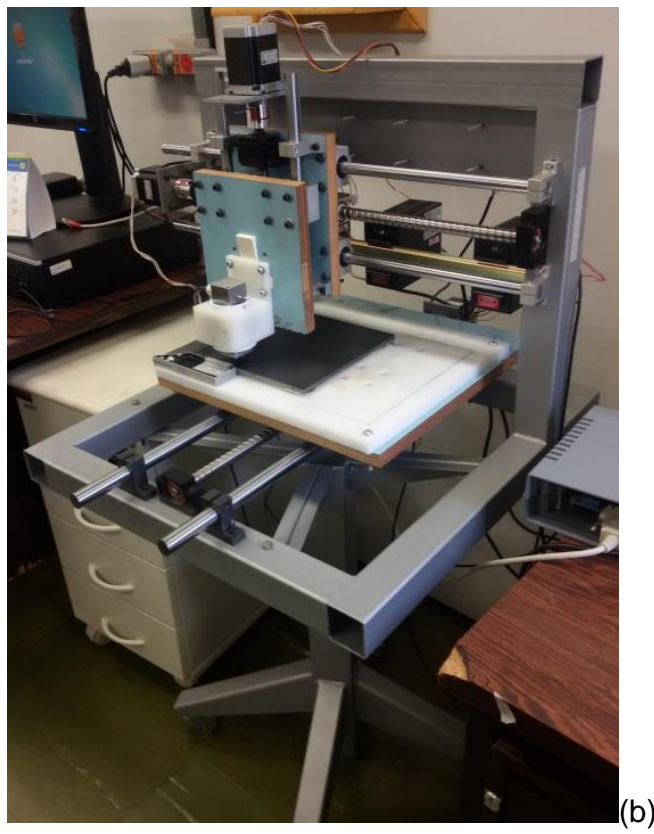

Figura 8. Projeto (a) e construção (b) do dispositivo de movimentação XYZ.

* Contribuição técnica ao $18^{\circ}$ Seminário de Automação e TI Industrial, 23 a 26 de setembro de 2014 , São Paulo, SP, Brasil. 
Para seu acionamento, cada motor de passo possui uma fonte de tensão contínua e um driver. O driver, por meio de sinais discretos de habilitação (EN), direção de rotação (DIR) e passo (STEP), enviados pelo computador, aciona o movimento do motor. Intermediando a conexão entre o computador e os três drivers há uma placa isoladora.

\section{RESULTADOS E DISCUSSÃO}

Foram realizados testes de trajetória e de medição no SAMRMB.

\subsection{Teste de Trajetória}

Os testes de trajetória foram realizados configurando-se previamente a trajetória no "Plate Barkhausen" e, com o auxílio de uma caneta fixada ao suporte do sensor, os pontos de medição foram marcados em papel milimetrado. As linhas verdes tracejadas nas Figuras 9 e 10 indicam a trajetória configurada no "Plate Barkhausen" e os pontos em vermelho representam os pontos de medição pelo sensor durante o teste. Na Figura 9, são mostrados dois testes: uma trajetória em linha reta no eixo $X$ e outra no eixo $\mathrm{Y}$, ambas com espaçamento de $20 \mathrm{~mm}$ entre cada medida. Nota-se o correto posicionamento dos pontos em vermelho.

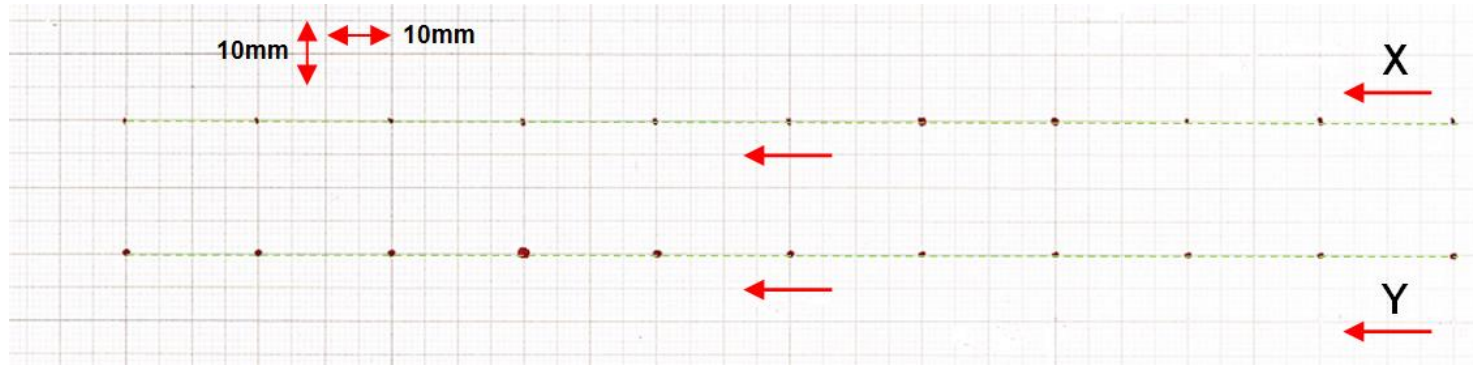

Figura 9. Testes de trajetória em apenas um eixo.

Na Figura 10, é mostrada a trajetória configurada como na Figura 5. Observa-se que a trajetória real (pontos em vermelho) possui um pequeno desvio em relação à trajetória esperada (linha tracejada em verde). O desvio está na direção $Y$ e é maior quanto maior for a distância percorrida na direção $X$. Isso ocorre porque os eixos $X e$ $Y$ do dispositivo $X Y Z$ não são totalmente perpendiculares, devido ao desalinhamento na montagem dos mesmos. Esse problema pode ser desconsiderado quando se usa uma trajetória com deslocamento apenas em um eixo (Figura 9).

* Contribuição técnica ao $18^{\circ}$ Seminário de Automação e TI Industrial, 23 a 26 de setembro de 2014, São Paulo, SP, Brasil. 


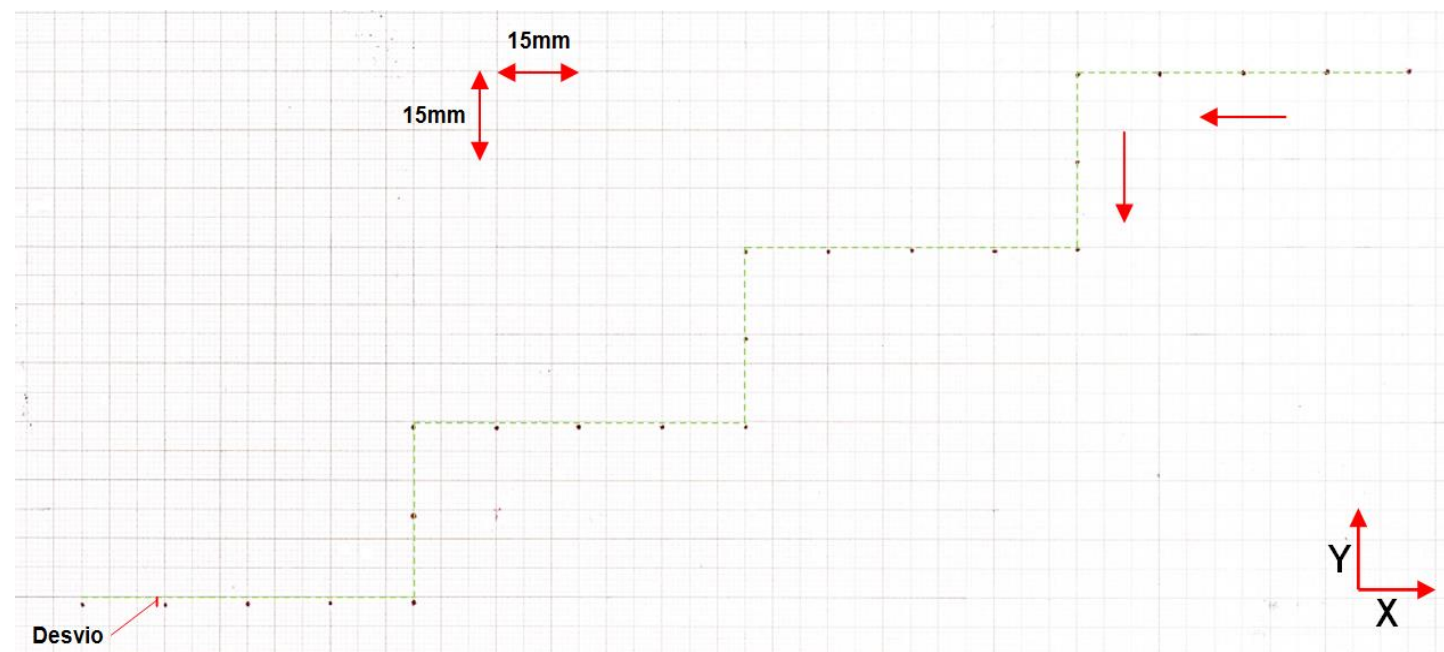

Desvio

Figura 10. Teste de trajetória em ambos os eixos.

Foi feito ainda um teste de repetibilidade de trajetória no qual uma trajetória foi repetida três vezes em "vai e vem". Foi verifica a coincidência das marcações da caneta em cada ponto, o que mostra a repetibilidade da trajetória.

\subsection{Teste de Medição}

Para o teste da medição de RMB e do cálculo do valor RMS, foi montado um experimento no qual várias tensões mecânicas foram aplicadas a um corpo de prova metálico e os respectivos sinais magnéticos Barkhausen medidos com o "Plate Barkhausen" e com o "Microscan 600". Na Figura 11, pode ser visto o gráfico que relaciona a tensão mecânica à qual o corpo de prova é submetido e o valor RMS do RMB medido por ambos os softwares. Podemos constatar pela observação da figura que as curvas do "Microscan 600" e do "Plate Barkhausen" não são coincidentes. Isso é esperado, pois o processamento ao qual os dados são submetidos é diferentes nos dois softwares. Contudo, as duas curvas apresentam a mesma forma, o que é satisfatório.

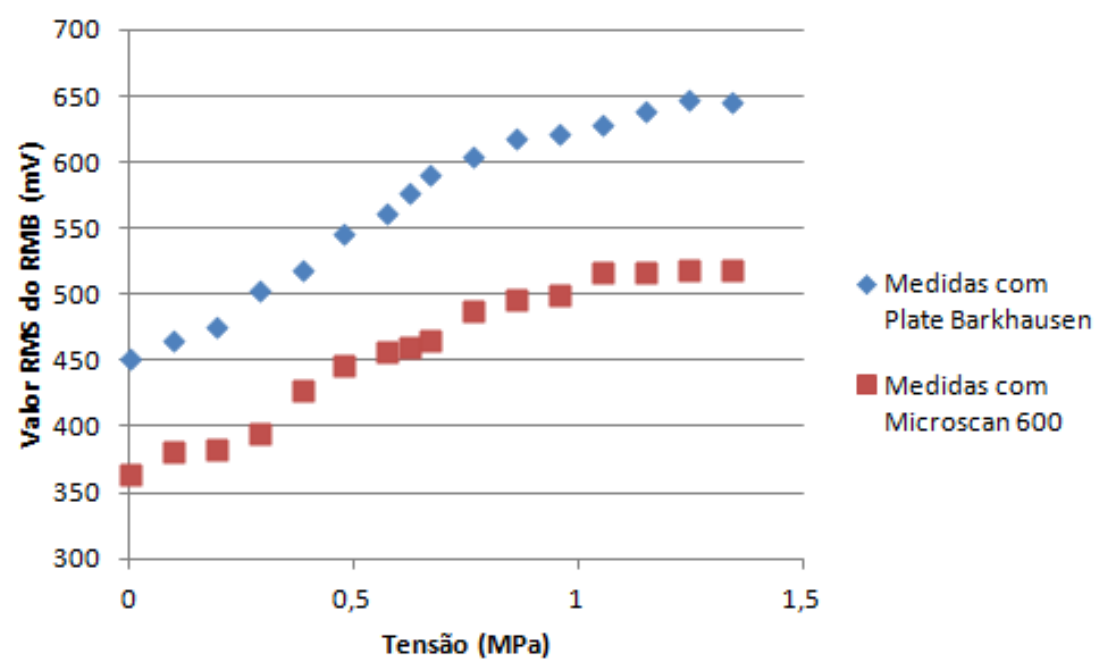

Figura 11. Gráfico que relaciona a tensão mecânica à qual o corpo de prova é submetido e o valor RMS do RMB medido com o "Microscan 600" e com o "Plate Barkhausen". Parâmetros usados: Voltagem de Magnetização $1.5 \mathrm{Vpp}$. Freqüência de Magnetização $125 \mathrm{~Hz}$ e Frequência de Amostragem $2.5 \mathrm{MHz}$.

* Contribuição técnica ao $18^{\circ}$ Seminário de Automação e TI Industrial, 23 a 26 de setembro de 2014, São Paulo, SP, Brasil. 


\section{CONCLUSÃo}

Neste trabalho, é apresentado o Sistema Automático de Medição de Ruído Magnético Barkhausen (SAMRMB) para a execução de medidas únicas ou batelada em chapas.

O software "Plate Barkhausen" realiza a medição, aquisição das amostras e cálculo do valor RMS com sucesso. Juntamente com o dispositivo de movimentação $X Y Z$, executa a trajetória e realiza o posicionamento do sensor corretamente, exceto por um pequeno desalinhamento entre os eixos $\mathrm{X}$ e $\mathrm{Y}$.

\section{REFERÊNCIAS}

1 Sipahi LB. Overview of applications of micromagnetic Barkhausen emissions as noninvasive material characterization technique. Journal of Applied Physics. mai 1994; 75:6976- 6980.

2 Kishimoto ET, Moraes, JCT. Qual a contribuição da automação para um laboratório de ensaios?. In: Congresso da Qualidade Em Metrologia; jun 2008; São Paulo. Disponível em: http://www.vertent.net/remesp/enqualab2008/cdrom/pdf/TT038.pdf.

3 Spinrad RJ. Automation in the Laboratory. Science. out 1967; 7:55-60.

4 Fontana AA. Sistema de controle automático de posição para telescópios e rastreamento de objetos astronômicos [monografia conclusão de curso]. Vitória: Universidade Federal do Espírito Santo, Centro Tecnológico; 2005. Disponível em: http://www2.ele.ufes.br/ projgrad/documentos/PG2005_1/angeloaugustofontana.pdf.

5 Sato $\mathrm{FH}$. Automação do sistema de carregamento em ensaios estruturais [monografia conclusão de curso]. Ilha Solteira: Universidade Federal Paulista, Faculdade de Engenharia de llha Solteira; 2001. Disponível em: http://www.ppgec.feis.unesp.br/teses/2002/sato.pdf.

6 Serna-Giraldo CP. Avaliação de juntas soldadas de aços carbono através do ruído magnético Barkhausen [tese doutorado]. São Paulo: Universidade de São Paulo; 2007.

* Contribuição técnica ao $18^{\circ}$ Seminário de Automação e TI Industrial, 23 a 26 de setembro de 2014, São Paulo, SP, Brasil. 